\title{
A Modified Fatigue Damage Model for High-Cycle Fatigue Life Prediction
}

\author{
Meng Wang, ${ }^{1,2}$ Qingguo Fei, ${ }^{1,2}$ and Peiwei Zhang ${ }^{1,2}$ \\ ${ }^{1}$ Department of Engineering Mechanics, Southeast University, Nanjing 210096, China \\ ${ }^{2}$ Jiangsu Key Laboratory of Engineering Mechanics, Nanjing 210096, China \\ Correspondence should be addressed to Qingguo Fei; qgfei@seu.edu.cn
}

Received 15 November 2015; Revised 17 January 2016; Accepted 20 January 2016

Academic Editor: Konstantinos G. Anthymidis

Copyright (c) 2016 Meng Wang et al. This is an open access article distributed under the Creative Commons Attribution License, which permits unrestricted use, distribution, and reproduction in any medium, provided the original work is properly cited.

\begin{abstract}
Based on the assumption of quasibrittle failure under high-cycle fatigue for the metal material, the damage constitutive equation and the modified damage evolution equation are obtained with continuum damage mechanics. Then, finite element method (FEM) is used to describe the failure process of metal material. The increment of specimen's life and damage state can be researched using damage mechanics-FEM. Finally, the lifetime of the specimen is got at the given stress level. The damage mechanics-FEM is inserted into ABAQUS with subroutine USDFLD and the Python language is used to simulate the fatigue process of titanium alloy specimens. The simulation results have a good agreement with the testing results under constant amplitude loading, which proves the accuracy of the method.
\end{abstract}

\section{Introduction}

Fatigue failure, as one of the major causes of damage for the mechanical components, relates to alternative loads subjected on engineering structures, which drew increasingly researching work focusing on the fatigue life prediction. There are two methods to forecast the structure fatigue life: one is fatigue safe life prediction based on fatigue cumulative damage theories and the other is damage tolerance method based on fracture mechanics [1]. In general, the fatigue life of material is estimated based on the damage tolerance method. However, a high proportion of the overall lifetime for the metal high-cycle fatigue is the fatigue initiation life [2].

Damage mechanics is the theory studying the mechanisms and rules of solid material degradation performance by changing mechanical variables under the external loads. As an important branch of damage mechanics, the continuum damage mechanics [3-5] is a theory based on continuum mechanics and irreversible thermodynamics. Therefore, the process of fatigue crack initiation and development can be analyzed by the fatigue life prediction model based on continuum damage mechanics. And the model of fatigue damage can be classified briefly into ductile fatigue damage model [6] and brittle fatigue damage model [7, 8]. Considering that the metal subjected to high-cycle fatigue could be regarded as quasibrittle material, the brittle damage mechanism is often employed to build a damage propagation model on the basis of irreversible thermodynamics [8], which is broadly accepted in engineering practices on account of less parameters of evolution equations. Moreover, the damage numerical analysis method is another core element of damage mechanics. The determination of damage field and the forecast of fatigue life have been achieved by the damage mechanics-finite element method [9-11], which can be utilized to obtain the lifetime of damage initiation and damage propagation path.

In this study, a brittle fatigue damage model is modified and combined with a damage constitutive model to predict the fatigue life of the metal material in Section 2. The method to estimate fatigue lifetime of titanium alloy components is accomplished by the commercial software ABAQUS which is developed by programming in Python and Fortran in Section 3. Then, the correctness of the fatigue damage model, the finite element model, and the simulation method is 
validated through comparing the analytical results with experimental results under constant amplitude loading in Section 4.

\section{Modified Fatigue Damage Model}

The degeneration of material mechanical properties results from nucleation, growth, and integration of microcracks and voids within metal material. Thus, for one-dimensional structure, damage variable $[3,12]$ can be defined as

$$
D=\frac{S_{D}}{S},
$$

where $S$ is the total area of the cross section and $S_{D}$ is the total area of microcracks and cavities.

Since Lemaitre [4] has obtained the true stress of material by the strain equivalence theory after damage initiation, the constitutive relation of material can be defined, under the state of one-dimensional stress, as

$$
\varepsilon=\frac{\widetilde{\sigma}}{E_{0}}=\frac{\sigma}{E_{0}(1-D)},
$$

where $\widetilde{\sigma}, E_{0}$, and $\varepsilon$ are, respectively, the true stress of damaged material, the initial elasticity modulus of material, and the elastic strain.

The constitutive relation of three-dimensional isotropic damage material can be written as

$$
\sigma_{i j}=C_{i j k l}(1-D) \varepsilon_{k l} \text {, }
$$

where $D=0$ means the material is undamaged and $D=1$ means the material has completely failed.

Here, a damage evolution model $[8,13]$ is introduced as

$$
\frac{d D}{d N}=\frac{\bar{B}}{q(1-D)^{2 q}}\left(\sigma_{\mathrm{eqM}}^{2 q}-\sigma_{\mathrm{eqm}}^{2 q}\right),
$$

where $\bar{B}$ and $q$ are both material constants, $\sigma_{\text {eqM }}$ is the maximum equivalent stress of loading (MISES), and $\sigma_{\text {eqm }}$ is the minimum equivalent stress.

To obtain the stress and damage field of the model numerically, the nonlinear equations above are solved with the damage parameter as the increment variable. According to (3), $C_{i j k l}(1-D)$ is a constant matrix in the increment step of damage parameter. So, the damaged material can be considered as a "new" kind of undamaged material. For the linear elastic materials,

$$
\sigma_{\text {eqm }}=R \sigma_{\text {eqM }}
$$

where $R$ is the stress ratio of fatigue loads and a constant during the damage process.

Rewrite (4) into

$$
\frac{d D}{d N}=\frac{\bar{B}\left(1-R^{2 q}\right)}{q(1-D)^{2 q}} \sigma_{\mathrm{eqM}}^{2 q}
$$

where $\bar{B}$ and $q$ are the material constants in this study. Equation (6) is modified as follows:

$$
\frac{d D}{d N}=\frac{\sigma_{\mathrm{eqM}}^{2 q}}{B(1-D)^{2 q}} .
$$

Since the stress ratio $R$ is constant during the modeling cycle, $B=q / \bar{B}\left(1-R^{2 q}\right)$ is a constant too. The modified model has less fitting parameters and is accommodative to be integrated into finite element programs.

Combined with geometric equation, constitutive relation, equilibrium equation, and boundary conditions, the damage evolution model modified above can be applied to compute the lifetime based on known conditions of stress and damage. The lifetime estimation of damage initiation and propagation is accomplished by the damage mechanics-finite element method.

\section{Numerical Methods of Fatigue Life Prediction}

Damage mechanics-finite element method [10, 13] is employed for the fatigue life prediction and damage field simulation. The main feature of the method is that the damage increment of dangerous point is kept constant in the analytical process. The steps could be described as follows:

(1) Finite element model considering damage is developed. At the first loading step, the values of damage of the elements are assumed to be zero.

(2) Damage evolution is calculated according to the stress field, and the element with a maximum damage value is selected as the dangerous point of the structure. Furthermore, the damage status of the dangerous point needs to be checked during every loading step, and if it has failed, the dangerous point of the structure should be reestimated.

(3) Life increment of the $i$ th modeling cycle $(i \geq 2)$ is calculated according to (7):

$$
\Delta N_{i}=\frac{B\left(1-D_{i-1}\right)}{\sigma_{\text {eqM }}^{2 q}} \Delta D .
$$

The value of total life is updated as

$$
N_{i}=\sum_{0}^{j=i} \Delta N_{j}
$$

(4) The damage increment of gauss points of other elements is obtained on the basis of life increment:

$$
\Delta D_{i}(x)=\frac{\left(\sigma_{i-1}(x)\right)_{\mathrm{eqM}}^{2 q}}{B\left(1-D_{i-1}(x)\right)^{2 q}} \Delta N_{i} .
$$

The damage values of gauss integral points of each element are

$$
D(x)_{i}=\sum_{0}^{j=i} \Delta D(x)_{j} .
$$




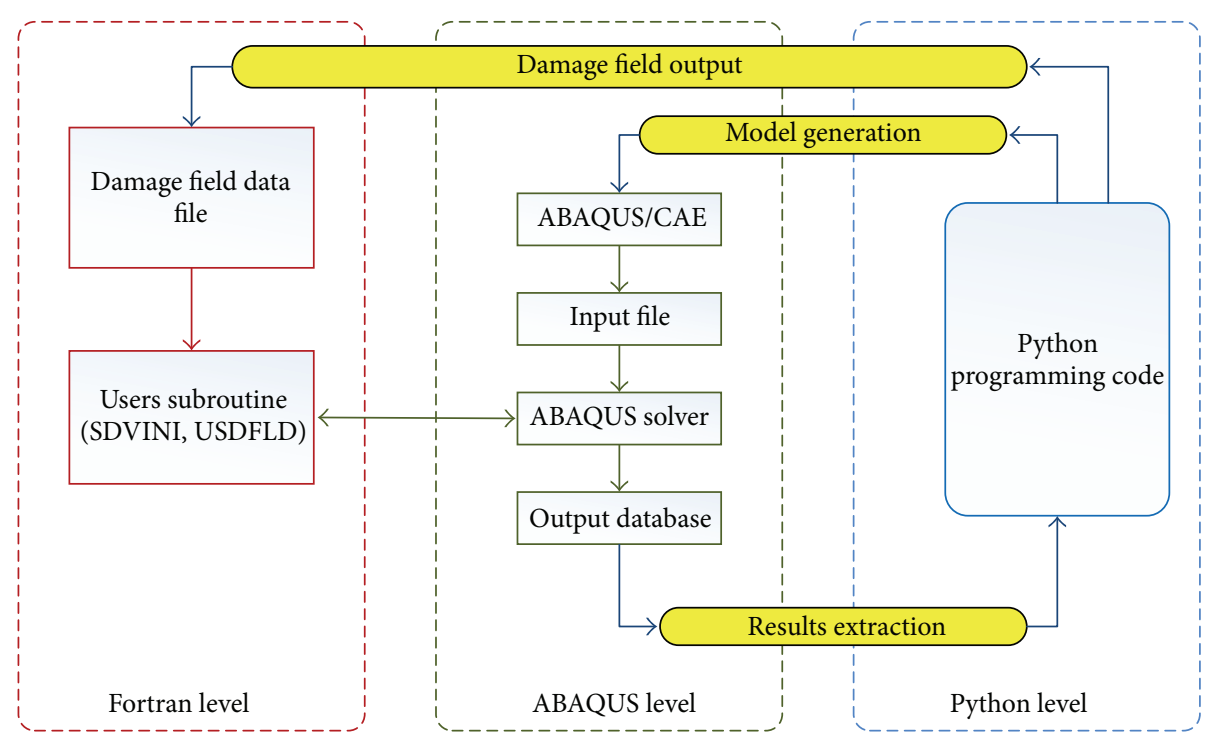

FIgURE 1: Numerical scheme for damage mechanics-finite element method.

(5) With the new damage field, the stress field is updated until the components lose efficacy or $\Delta N_{i}$ is infinitesimally smaller than $\Delta N_{i-1}$.

The damage mechanics-finite element method is implemented in ABAQUS, coding with Python and Fortran to use the built-in functions, such as finite element modeling, postprocess of stress, and damage field analysis results. The implementation flow chart of damage mechanics-finite element method is shown in Figure 1.

(1) Firstly, finite element model is built using the Python language in ABAQUS and at the same time the initial value of damage field is set to zero.

(2) Secondly, the damage field is imported by a subroutine named SDVINI and passed to another subroutine named USDFLD. After that, the structural stress will be analyzed.

(3) Thirdly, after the stress distributions of the inner structure are obtained, Python language is used to read the results, confirm the dangerous points of structure, obtain the life increment and damage values of other gauss integral points considering the damage increment, and output the file containing damage status of the model.

(4) Fourthly, the dangerous point needs to be reselected if the damage value of dangerous element reaches a critical state, and step (2) is conducted until the component loses efficacy.

\section{Numerical Investigation}

The high-cycle fatigue life of standard test specimen of TC4 alloy is analyzed using finite element program with damage mechanics calculation method implemented in ABAQUS. The simulation results are validated through comparing them with the fatigue test results in Practical Handbook of Engineering Materials [14]. Then, the fatigue life of notched specimen is predicted according to the damage evolution model with parameters verified by experimental results of smooth specimens.

4.1. Parameter Fitting. To improve the calculating efficiency, axisymmetric model subjected to uniformly distributed loads is constructed with elastic modulus of material $116 \mathrm{GPa}$, and Poisson's ratio 0.3 .

Parameters $q$ and $B$ of damage propagation formula are obtained by fitting the damage evolution model through the fatigue test results of standard smooth test specimens of TC4 alloy in Practical Handbook of Engineering Materials [14].

For the smooth components $\left(K_{T}=1\right): \sigma_{\text {eq }}=|\sigma|$, von Mises stress almost equals tensile stress. Combination of the test results of the standard smooth test pieces of TC4 alloy and closed-form solution method of fatigue life prediction [15], with the integral of (6), is

$$
\int_{0}^{1}(1-D)^{2 q} d D=\frac{\sigma_{\mathrm{eqM}}^{2 q}}{B} \int_{0}^{N_{f}} d N ;
$$

$q=14$ is derived according to the test results.

The value of damage increment $\Delta D$ is required by damage mechanics-finite elements algorithm and determined appropriately to reduce the computing time [16]. The value of $\Delta D$ as 0.3 is adopted.

During the process of data fitting, parameter $B$ is found to be dependent on experimental data chosen largely. According to the general rules of metal damage evolution and symmetrical stress of cross section of smooth specimen, an assumption [17] is made that a high proportion of the component fatigue 


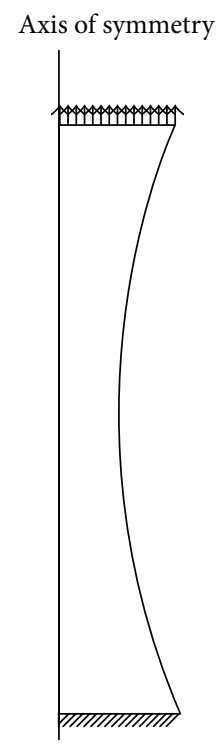

FIGURE 2: Boundary condition.

life corresponds to the first dangerous point failure life and then the other elements will lose efficacy rapidly after failure. Thus, according to (7), with the fitting formula listed as

$$
N=\left(\sigma_{\text {eqM }}^{(-2 q)}\right) B
$$

parameter $B$ is $6 \times 10^{46}$, after choosing a test life of a certain stress level.

4.2. The Fatigue Life Simulation of Smooth Specimens. Smooth specimens are analyzed by damage mechanics-finite element method in ABAQUS in this subsection. The value of nominal maximum stress of specimen section is $520 \mathrm{MPa}$. Loading and boundary conditions of tensile-tensile fatigue standard samples in accordance with HB 5287-96 and [14] are shown in Figure 2.

As shown in Figure 3, in order to simplify the models, the symmetric models are utilized to calculate the stress distribution under the conditions of different damage state at the first dangerous point.

In Figure 3, different colors demonstrate that the areas are in different stress states with the damage value increasing at the dangerous point. When the damage value of the first dangerous point reaches 0.3 , the increment of fatigue life (the damage value of the dangerous point increases from 0 to 0.3 ) is 3989000 . And the relative life increment is 149700 , when damage value of dangerous point jumps from 0.3 to 0.6 . The increment is 1361, when the damage value increases from 0.6 to 0.9. After failure of the first dangerous point, the rapid decline of bearing area of component (from $D_{\max }=0.9$ to $D_{\text {max }} \rightarrow 1$ ) illustrates that component has totally failed and the corresponding life increment is small, which proves the correctness of the assumption made in Section 4.1. As shown in Table 1, fatigue life can be predicted by damage mechanicsFEM under the other loading conditions, the analysis error of which satisfies the engineering requirement.
TABLE 1: The fatigue life prediction results of smooth specimen.

\begin{tabular}{lccc}
\hline Maximum stress (MPa) & Test life & Forecast life & Error \\
\hline 520 & 3011083 & 4140061 & $37.48 \%$ \\
550 & 1331951 & 1819000 & $36.6 \%$ \\
570 & 829118 & 1103000 & $33.03 \%$ \\
\hline
\end{tabular}

4.3. The Fatigue Simulation of Notched Specimens. The material parameters confirmed by smooth specimens test results are used to estimate the fatigue life of the notched specimens. Axial symmetry model is adopted in order to improve calculation efficiency. Moreover, finite element mesh is refined to increase the accuracy of stress states around the notch of specimen.

Simulation fatigue tests are performed under the conditions that the stress concentration factor $K_{t}$ of specimen is 3 in the Practical Handbook of Engineering Materials [14] and the nominal maximum stress of the notched specimen equals $720 \mathrm{MPa}$. The size of the model is in accordance with HB 5287-96. Stress changings are shown in Figure 4, when the damage increment value equals 0.3 and the number of analyzing modeling loop $(n)$ equals 10,20 , and 30 , separately. The total life increment of three statistics processes (from the beginning to $n$ ) is 1594457,2016702 , and 2182710 separately. And the relative life increment (from the former statistics to $n)$ is 1594457,422245 , and 166008 separately.

Similar to those in Figure 3, the elements with higher stress represent that the elements are under stress concentration condition. On the contrary, the elements behind the notch with lower stress values denote that the elements have fully failed. With the increasing of loading cycles, the area with lower stress states will extend from the notch region to the inner part. After cycles of loading, the extending speed of failure area increases faster. The failure life of first dangerous element of notched specimen is 371600 during the analysis process of the first 10 modeling cycles. Therefore, compared to the total fatigue life of the notched specimen, damage evolution life in the elements has a higher proportion during the failing process. It could be found that the relative life increment of first analyzing modeling loop is at least one order of magnitude greater than life increment during the subsequent same number of modeling cycles. With failure units increasing, both less bearing area and higher stress state at the dangerous points contribute to the increase of failure rate. The life increment of the third modeling cycles is much smaller than that of the second modeling cycles, so the life with crack extending to $0.5 \mathrm{~mm}$ can be used to forecast the final life of components. The process of simplification can forecast an acceptable fatigue life and reduce the computing time.

The fatigue life prediction results of notched specimen subjected to other nominal stresses are shown in Figure 5. And the fatigue life of components is analyzed under the other external loading conditions, the results of which are similar to the experimental results and acceptable to engineering taking into account the dispersion of fatigue life. 


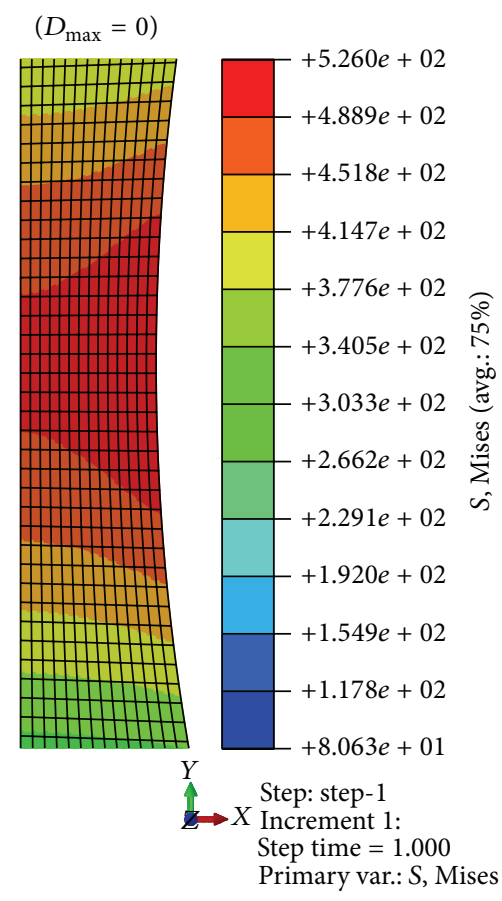

$\left(D_{\max }=0.9\right)$

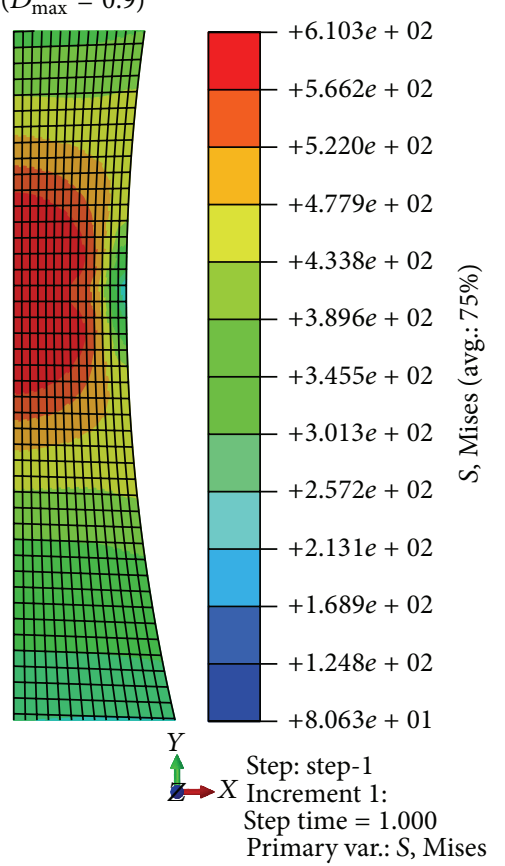

$\left(D_{\max }=0.3\right)$

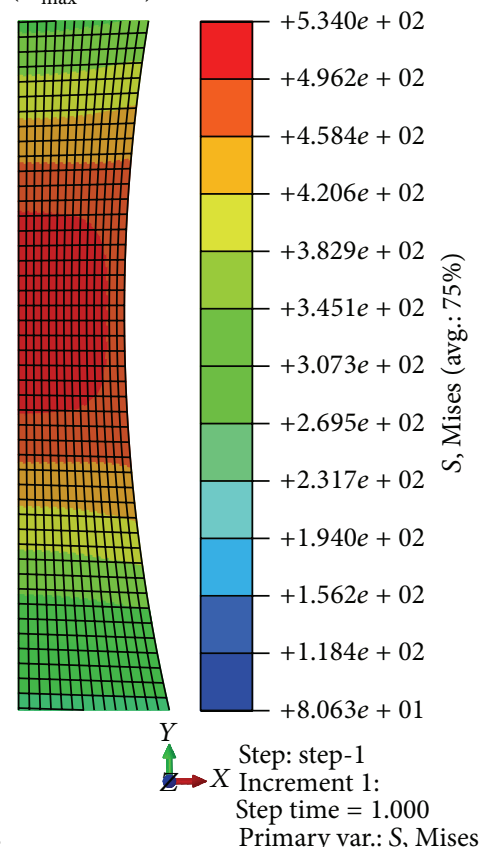

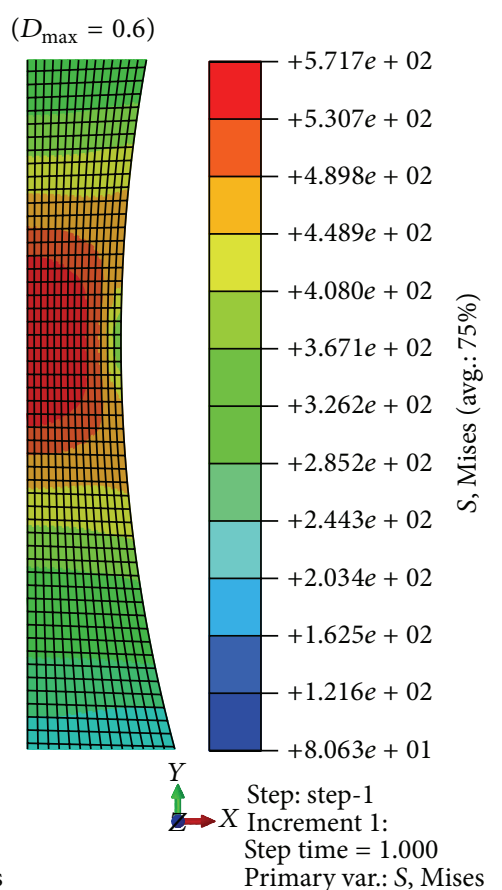

$\left(D_{\max } \rightarrow 1\right)$

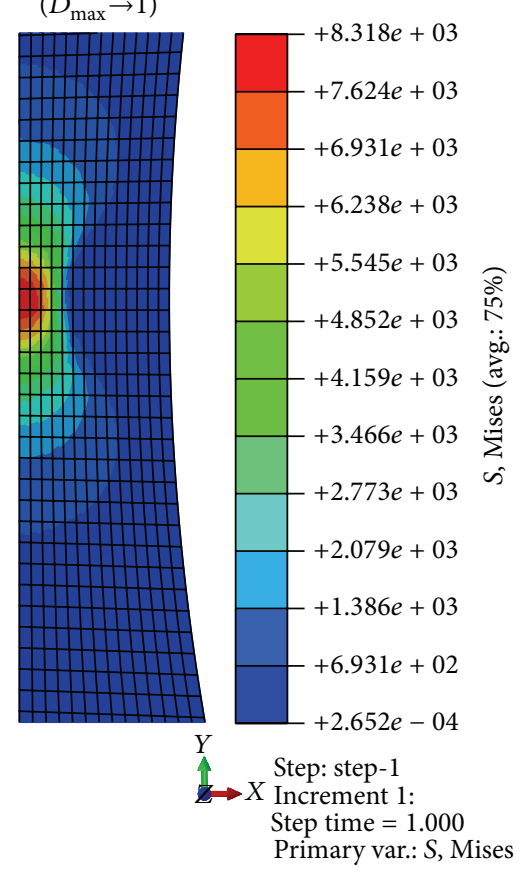

FIGURE 3: Stress changing of smooth specimen during damage evolution.

\section{Conclusions}

To forecast the high-cycle fatigue life of metal material, constitutive relation of damage material and damage evolution relation can be employed by using the method of damage mechanics and introducing the damage variable under the supposition that metal material subjected to high-cycle load will be brittle fractured. And then, through the equilibrium equations, the fatigue life can be predicted. The damage evolution method is modified considering the damage numerical analysis method. After that, the damage mechanics-finite element method can be embedded into ABAQUS by the script and subroutine based on Python and Fortran.

Based on the assumed distribution regularities of smoothed specimen fatigue life and damage rules (the longer life of components corresponds to the former damage growth, and other elements will lose efficacy rapidly after the failure of the first dangerous point), the parameters of damage evolution equations and damage increment are determined and the correctness of assumption has been verified by 

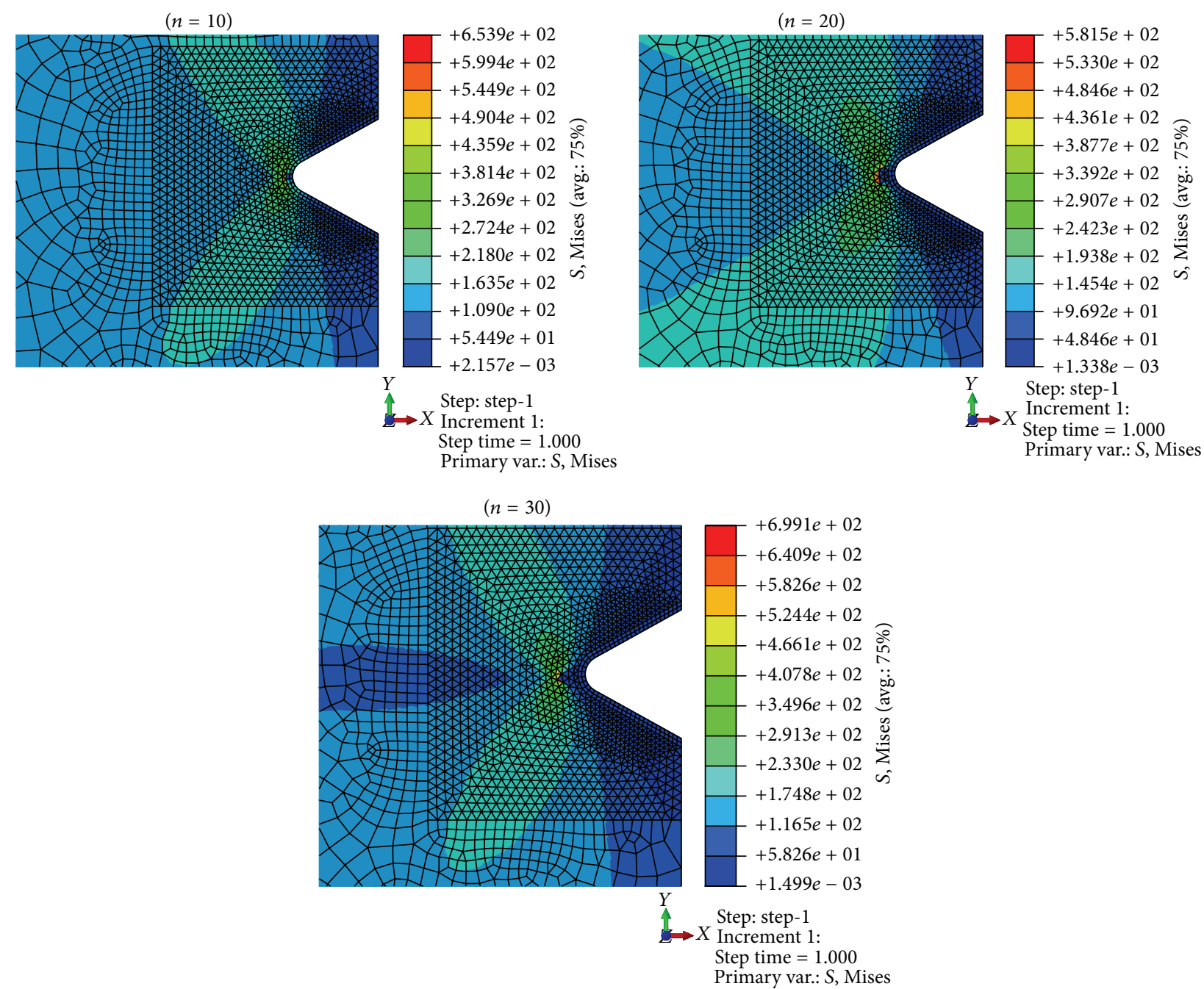

FIGURE 4: Stress changing of notched specimen during damage evolution.

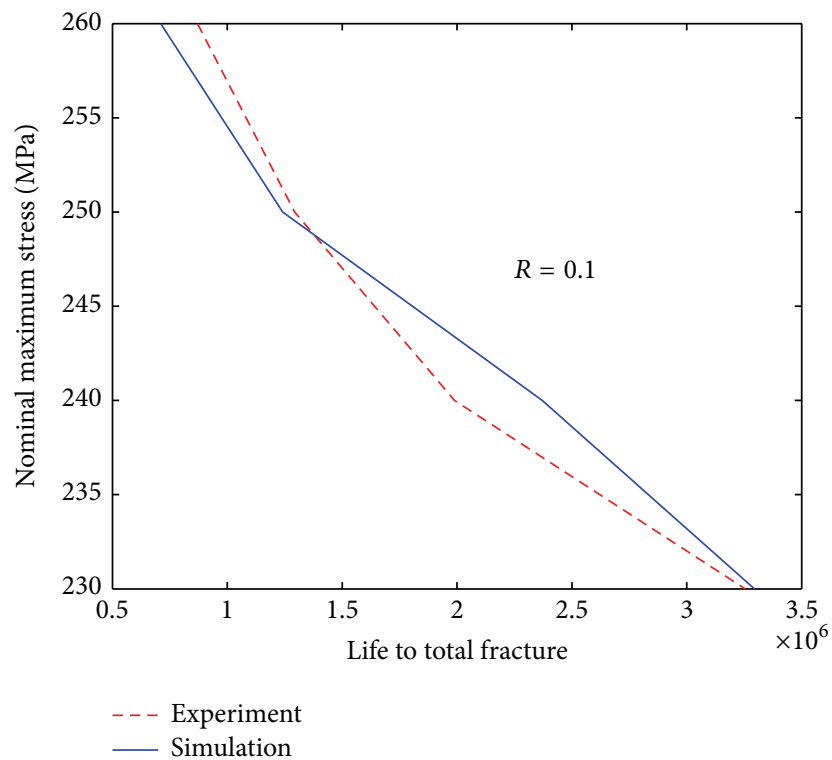

FIgURE 5: The fatigue life prediction results of notched specimen. analyzing the simulation results of the smooth specimens and comparing them with testing results under constant amplitude loading. The prediction of fatigue life of notched specimens under constant amplitude loading has been achieved by using parameters determined by the smooth specimens. The damage evolution paths of components and fatigue life results obtained demonstrate that the propagation of damage elements is an important process of damage failure in the notched specimens. At the same time, with the growth of damage elements, failure rate of the elements increases faster. The overall lifespan in the first ten models, compared with that of the following another ten models, is at least one order of magnitude greater. Thus, considering the dispersion, only the failure life of elements within $0.5 \mathrm{~mm}$ from the notch region is taken (the distance from the notch region to the units is $0.5 \mathrm{~mm}$ ) into account to predict the fatigue life accurately and reduce the computing time. This study not only presents the different failure process of the two components but also explains the rules of damage evolution in the two different samples. Besides, the simulation process 
is simplified based on the reasonable hypothesis and the analyzed results.

\section{Conflict of Interests}

The authors declare that there is no conflict of interests regarding the publication of this paper.

\section{Acknowledgments}

The presented study is supported by the National Natural Science Foundation of China (Grant nos. 11202050 and 11572086 and 11402052) and Jiangsu Natural Science Foundation of China (Grant nos. BK2012318 and BK20140616).

\section{References}

[1] J.-Z. Chen, L. Zhen, S.-J. Yang, and S.-L. Dai, "Effects of precipitates on fatigue crack growth rate of AA 7055 aluminum alloy," Transactions of Nonferrous Metals Society of China, vol. 20, no. 12, pp. 2209-2214, 2010.

[2] Z. Ange, Z. Jiucheng, and C. Mengcheng, Fatigue, Fracture and Damage, Xian Jiaotong University Press, Xian, China, 2006 (Chinese).

[3] J. L. Chaboche, "Continuum damage mechanics. Part Igeneral concepts," Journal of Applied Mechanics, vol. 55, no. 1, pp. 59-64, 1988.

[4] J. Lemaitre, A Course on Damage Mechanics, Springer, Berlin, Germany, 1992.

[5] J. Lemaitre and D. Marquis, "Modeling complex behavior of metals by the 'state-kinetic coupling theory"', Journal of Engineering Materials and Technology, vol. 114, no. 3, pp. 250-254, 1992.

[6] J. Lemaitre, "A continuous damage mechanics model for ductile fracture," Transactions of the ASME: Journal of Engineering Materials and Technology, vol. 107, no. 1, pp. 83-89, 1985.

[7] M. H. J. W. Paas, P. J. G. Schreurs, and W. A. M. Brekelmans, "A continuum approach to brittle and fatigue damage: theory and numerical procedures," International Journal of Solids and Structures, vol. 30, no. 4, pp. 579-599, 1993.

[8] Y.-C. Xiao, S. Li, and Z. Gao, "A continuum damage mechanics model for high cycle fatigue," International Journal of Fatigue, vol. 20, no. 7, pp. 503-508, 1998.

[9] X. Zhang, J. Zhao, and X.-D. Zheng, "Method of damage mechanics for prediction of structure member fatigue lives," in Handbook of Fatigue Crack Propagation in Metallic Structures, pp. 473-513, Elsevier, Philadelphia, Pa, USA, 1994.

[10] M. Zhang, Q. Meng, W. Hu, S. Shi, M. Hu, and X. Zhang, "Damage mechanics method for fatigue life prediction of PitchChange-Link," International Journal of Fatigue, vol. 32, no. 10, pp. 1683-1688, 2010.

[11] L. L. Sun, W. P. Hu, M. Zhang, and Q. C. Meng, "Damage mechanics-finite element method for vibrational fatigue life prediction of engineering structures with damping," Applied Mechanics and Materials, vol. 472, pp. 17-21, 2014.

[12] A. Warhadpande, B. Jalalahmadi, T. Slack, and F. Sadeghi, "A new finite element fatigue modeling approach for life scatter in tensile steel specimens," International Journal of Fatigue, vol. 32, no. 4, pp. 685-697, 2010.
[13] J. A. R. Bomidi, N. Weinzapfel, C.-P. Wang, and F. Sadeghi, "Experimental and numerical investigation of fatigue of thin tensile specimen," International Journal of Fatigue, vol. 44, pp. 116-130, 2012.

[14] Editorial Board, Engineering Materials Practical Handbook, vol. 4, version 2, Higher Education Press, Beijing, China, 2002 (Chinese).

[15] S. H. Stefanov, "Fatigue life prediction without cycle counting (by means of the integral method)," Journal of Theoretical and Applied Mechanics, vol. 32, no. 1, pp. 34-47, 2002.

[16] M. Naderi, S. H. Hoseini, and M. M. Khonsari, "Probabilistic simulation of fatigue damage and life scatter of metallic components," International Journal of Plasticity, vol. 43, pp. 101-115, 2013.

[17] V. V. Bolotin and I. L. Belousov, "Early fatigue crack growth as the damage accumulation process," Probabilistic Engineering Mechanics, vol. 16, no. 4, pp. 279-287, 2001. 

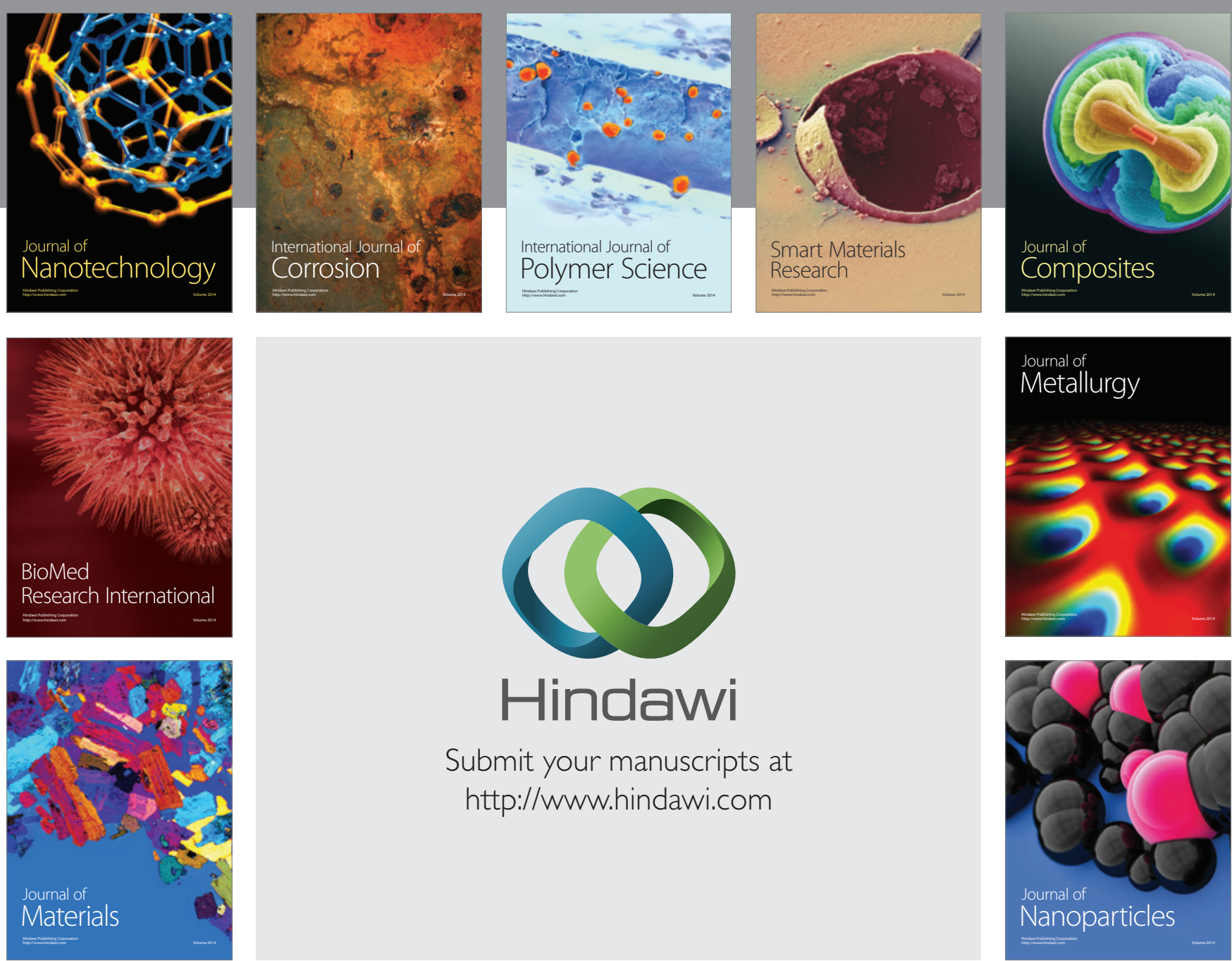

\section{Hindawi}

Submit your manuscripts at

http://www.hindawi.com

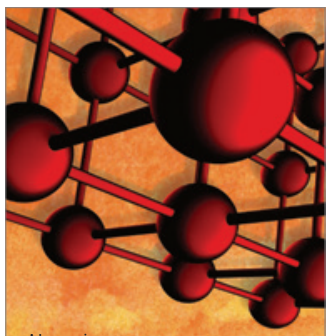

Materials Science and Engineering
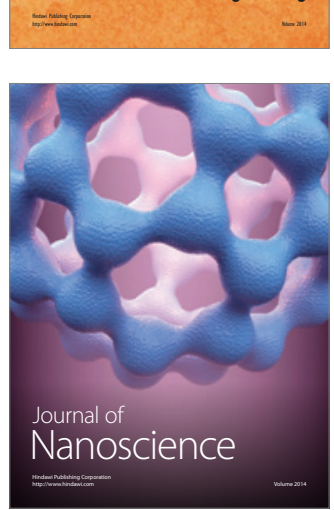
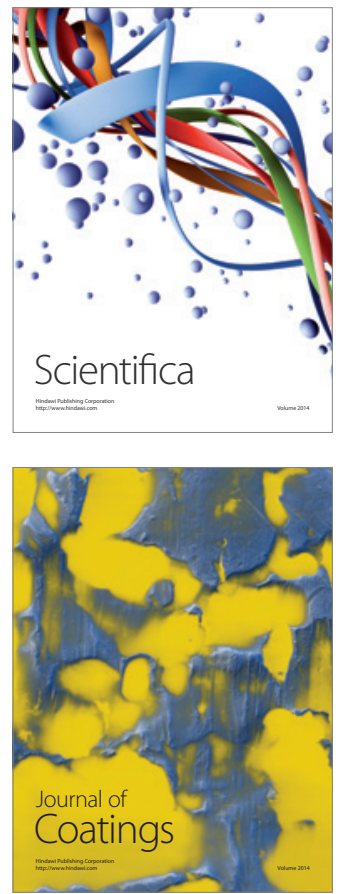
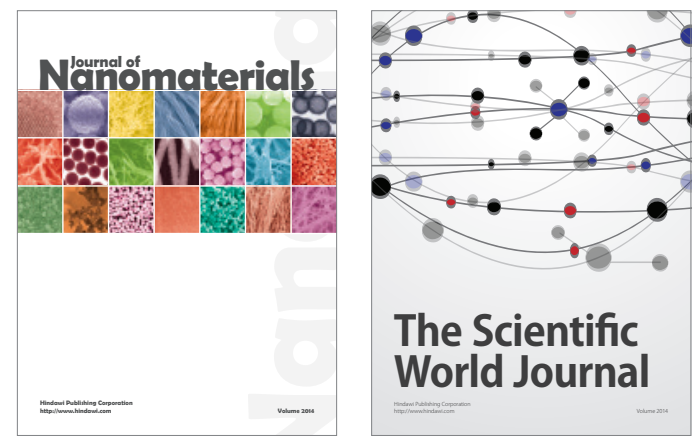

The Scientific World Journal
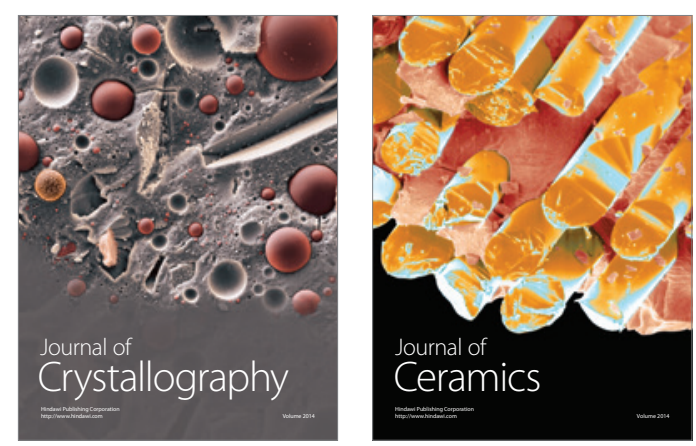
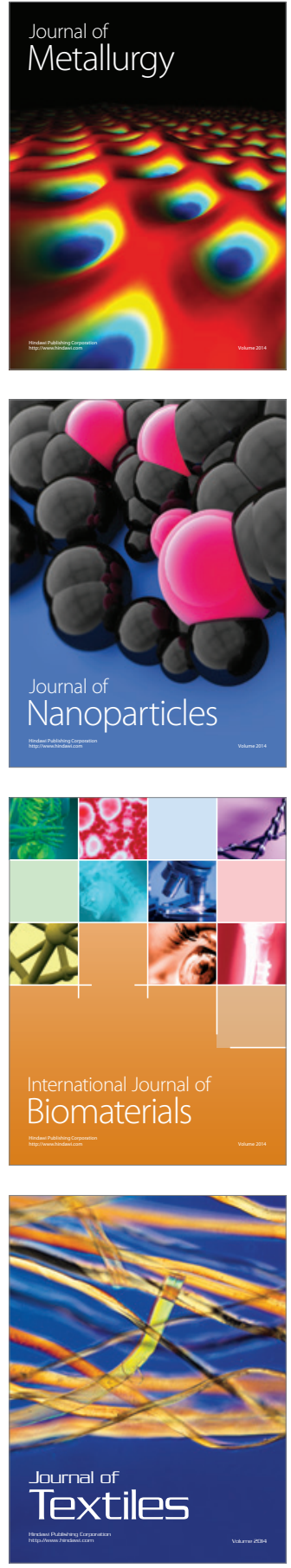